\title{
Description of Visfatin Adipokine and its Roles on Inflammation and Coronary Heart Disease: A Review Study
}

\author{
Assist. Prof.Dr. Wijdan Rajh Hamza Al-Kraity ${ }^{1 *}$; Ali Hussein Faisal ${ }^{2}$; Ali Nabeel Khaleel ${ }^{3}$; Mahmoud \\ Tuama Naeaam ${ }^{4}$; Ali Sattar Jabar ${ }^{5}$ \\ 1;2;;4;:5 Department Medical Laboratories Techniques, Al-Toosi University College, Iraq \\ *e-mail: dr.wijdan_rajh@altoosi.edu.iq
}

\begin{abstract}
Coronary heart disease (CHD) is a generic designation for a group of related syndromes resulting from myocardial ischemia - an imbalance between cardiac blood supply (perfusion) and myocardial oxygen demand. Visfatin (VF) is a recently discovered adipokine with different functions, Visfatin is mainly found in visceral adipose tissue and mimics insulin in lowering plasma glucose levels and, Visfatin emerges as a player in the development and progression of atherosclerotic lesions by directly promoting smooth muscle cell proliferation, Aberrant angiogenesis is now considered a feature of the atherogenic process in both coronary and carotid diseases. This adipokine was previously known as a pre-B-cell colony-enhancing factor (PBEF) or Nicotinamide phosphoribosyltransferase (NAmPRTase or Nampt) an enzyme that in humans is encoded by the NAMPT gene and demonstrated to be an intracellular protein with a key enzyme role in nicotinamide adenine dinucleotide (NAD)
\end{abstract}

Keywords: Coronary heart diseases, Visfatin, adipokine, pre-B-cell colony-enhancing factor (PBEF)

Article Information

Received: January 2, 2021; Revised: : February 26, 2021; Online: : March 1, 2021

\section{INTRODUCTION}

Coronary heart diseases (CHD) are the most common cause of morbidity and mortality in most countries (Sebregts et al., 2000), also due to the significant impact of coronary heart disease, it is important for identifying the determinants of risk for developing this disease, coronary artery diseases are the generic designation for the three forms of cardiac diseases, i.e., angina pectoris \{unstable angina (UA), stable angina (SA) $\}$, sudden cardiac death and acute myocardial infarction (AMI), In most cases, the abnormal outcomes from inadequate blood flow resultant lead to the development of atherosclerosis causing in narrowing of the coronary arteries, thus, CHD is also often called coronary artery disease (CAD) or ischemic heart disease (IHD) $\}$ ( Rydén et al., 2007; Naveen, 2009).

Adipokines and proinflammatory molecules that affect cardiovascular functions through not only as endocrine manner, but also by autocrine and paracrine mechanisms (De-Clercq et al., 2008). Consequence ends up with cytokine-mediated and systemic inflammation and atherosclerosis, Various studies revealed association of visfatin with inflammation (Moschen et al., 2007), metabolic syndrome (Shaker et al., 2011), endothelial dysfunction (Dahl et al., 2007), atherosclerosis (Filippatos et al., 2010) and ischemia reperfusion injury (Mazaherioun et al., 2012; Filippatos et al., 2013)

Visfatin (VF) is a recently discovered adipokine with different functions, Visfatin is mainly found in visceral adipose tissue and mimics insulin in lowering plasma glucose levels (Fukuhara et al.,2005; Shaker et al., 2011). Visfatin, other than the fat tissue, is expressed by various other cells and tissues such as neutrophiles, liver, heart and muscles as well as accepted as a growth factor for the maturation of pro-cells of the B-lymphocytes (Kitani et al., 2003; Gürsoy et al., 2014 ).

This adipokine was previously known as pre-Bcell colony-enhancing factor (PBEF) or Nicotinamide phosphoribosyltransferase (NAmPRTase or Nampt) an enzyme that in humans is encoded by the NAMPT gene and demonstrated to be an intracellular protein with a key enzyme role 
in nicotinamide adenine dinucleotide (NAD) synthesis (Samal et al., 1994; Fukuhara et al.,2005).

\section{Visfatin}

\section{Definition and functions}

Visfatin is one of the newly discovered adipokines, which is 52 kilodalton $(\mathrm{kDa})$ protein, Visfatin was first described as pre-B cell colony enhancing factor( PBEF) and Nicotinamide phosphoribosyl transferase (NAMPT) because it is involved in nicotinamide adeninedinucleotide synthesis from nicotinamide (Matsuda et al., 2005), it has insulin-mimetic properties (Stofkova et al., 2010). Recently, PBEF was identified by Fukuhara et al. (2005), as visfatin a novel adipokine - a protein mediator secreted by fat cells (high levels of expression in visceral fat cells). However, the visceral fat is not the only tissue where Visfatin is expressed. Leucocytes of peripheral blood (Samal et al ., 1994) or adipose tissue macrophages (Curat et al., 2006), hepatocytes (Garten et al., 2010) or skeletal muscles (Costford et al., 2010) also participate in Visfatin production and may influence Visfatin plasma levels.

The product of Visfatin/PBEF gene was initially identified by Samal and his collegues in (1994) as a cytokine subsequently named pre-B-cell colony-enhancing factor (PBEF) that is expressed in lymphocytes of peripheral blood and plays a role in lymphocytes maturation and inhibition of neutrophil apoptosis (Jia et al., 2004)

Visfatin role in immunity was explained as its gene was expressed in lymphocyte cells and it also induced the production of interleukins such as IL-1B, IL-6 and Tumor necrosis factor which are pro-inflammatory cytokines and IL-1 $\alpha$ and IL-10 which are anti-inflammatory cytokines(Moschen et al., 2010; Olszanecka et al., 2011) and it had an important roles in metabolism, aging and inflammation (Wang et al., 2011).

CHD diseases lead to the release of inflammatory mediators from the damaged tissue into saliva and other biological fluid (Trivedi et al., 2012). There is a direct association between visfatin levels and increased cardiovascular disease (Filippatos et al., 2013) and it had a role in many pathophysiological processes that eventually lead to cardiovascular disease such as hypertension and atherosclerosis (Omer and Mahmood, 2016).

\section{Structure}

Visfatin is a $52-\mathrm{kD}$ a protein (Flier et al., 2007), is active as a dimer, with each monomer containing 491 amino acids in humans, This has been proved by the fact that visfatin/PBEF/Nampt has two active sites at the interface of the dimeric protein, suggesting that dimerization is essential for the catalytic activity of the enzyme (Wang et al., 2006). Each monomer contains $19 \beta$-strands and $13 \alpha$ helices and is organized into two structural domains (KIM et al., 2006). Show figure (1).

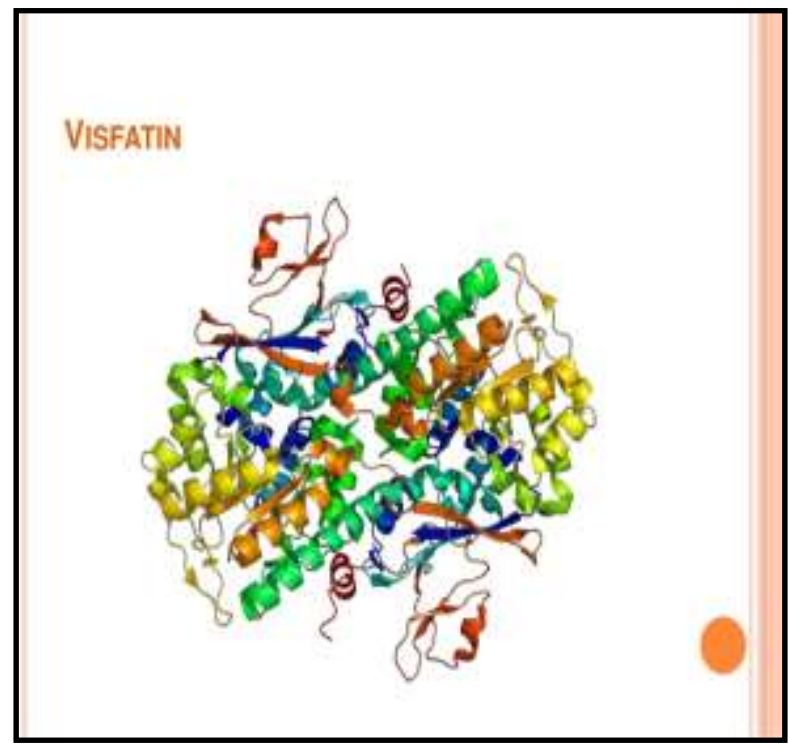

Figure (1): The crystal structure of visfatin adapted by (Raman, 2013).

The fragment-based identification of two novel and potent biochemical inhibitors of the nicotinamide phosphoribosyltransferase (NAMPT) enzyme is described in study of (Dragovich et al., 2014). These compounds (51 and 63) incorporate an amide moiety derived from 3-aminopyridine, and are thus structurally distinct from other known antiNAMPT agents. Each exhibits potent inhibition of NAMPT biochemical activity $\left(\mathrm{IC}_{50}=19\right.$ and $15 \mathrm{nM}$, respectively) as well as robust antiproliferative properties in A2780 cell culture experiments $\left(\mathrm{IC}_{50}=121\right.$ and $99 \mathrm{nM}$, respectively). Show the figure (2).

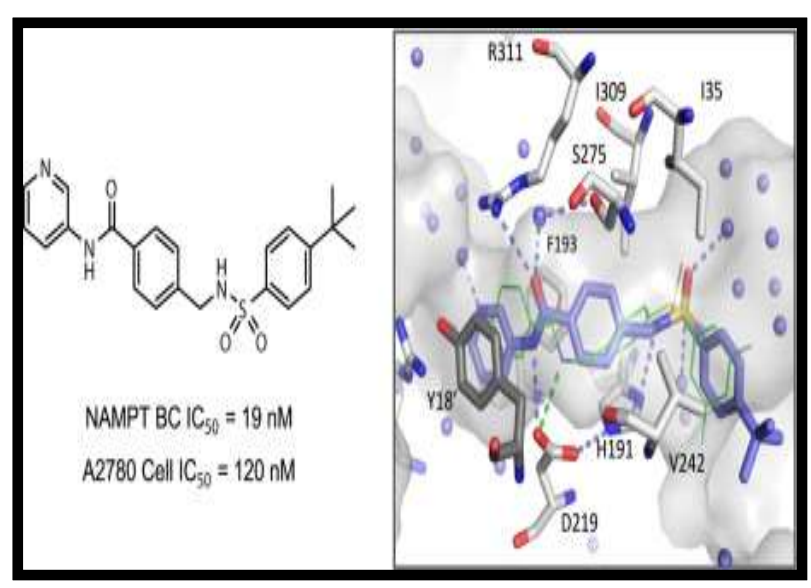

Figure (2): The chemical structure of visfatin, adapted by (Dragovich et al., 2014) 


\section{Action on cardiovascular systems}

\section{1-Cell proliferation and angiogenesis}

Proliferation of vascular smooth muscle cell (VSMC) is a hallmark of the development of atherosclerotic lesions. Importantly, perivascular visfatin/Nampt can act as a growth factor in vascular smoothmuscle cells, promoting cell proliferation in aortic smooth muscle cells though Nampt enzymatic activity (Wang et al., 2009).

Visfatin/Nampt emerges as a player in the development and progression of atherosclerotic lesions by directly promoting smooth muscle cell proliferation, Aberrant angiogenesis is now considered a feature of the atherogenic process in both coronary and carotid diseases (Moulton, 2006), In this line, it has also been described that VEGF synthesis and secretion as well as the expression of the VEGF receptor 2, which has been proposed to mediate the angiogenic actions of VEGF (Adya et al., 2008).

Besides VEGF, visfatin/Nampt upregulates the production of other proangiogenic soluble factors, such as fibroblast growth factor-2 (FGF-2), MCP-1, and IL-6, in endothelial cells (Lee et al., 2009). Indeed, both MCP-1 and FGF-2 have also been identified as mediators of visfatin/Nampt-induced angiogenesis (Bae et al., 2009). Show figure (3).

\section{Inflammation, atherogenesis and coronary heart disease.}

Growing scientific evidence supports that visfatin/Nampt can directly promote vascular inflammation by activating different cell types including endothelial cells and vascular smooth muscle cells. Moreover, visfatin/Nampt can also contribute to vascular inflammation through its immunomodulatory properties on immune cells (Moschen et al., 2007). Thus, visfatin/Nampt can exert direct actions onmonocytes. Hence, visfatin/Nampt promotes the synthesis and release of proinflammatory cytokines, such as tumor necrosis factor- $\alpha$ (TNF- $\alpha) \quad \alpha$ and IL-8, by peripheral mononuclear cells (Dahl et al ., 2007)

Additionally, visfatin/Nampt promotes macrophage survival (Li et al., 2008) which may help perpetuating vascular inflammation. In cultured

\section{CONCLUSION}

The visfatin is consider as a inflammatory markers that related with obesity and atherosclerosis, endothelial dysfunction and coronary heart disease. human vascular smooth muscle cells, our group demonstrated for the first time that visfatin/Nampt could directly exert inflammatory effects. Thus, exogenous administration of visfatin/Nampt activates ERK $1 / 2$ and NF- $\kappa \mathrm{B}$, resulting in enhanced expression of the inducible nitric oxide synthase (iNOS) (Romacho et al., 2009), The iNOS is a proinflammatory enzyme contributing to dysregulated NO production and subsequent peroxynitrite formation, Thus, iNOS induction plays a key role in endothelial dysfunction and vascular injury in diabetes-related vascular complications (Pacher et al., 2005). Show figure (3).

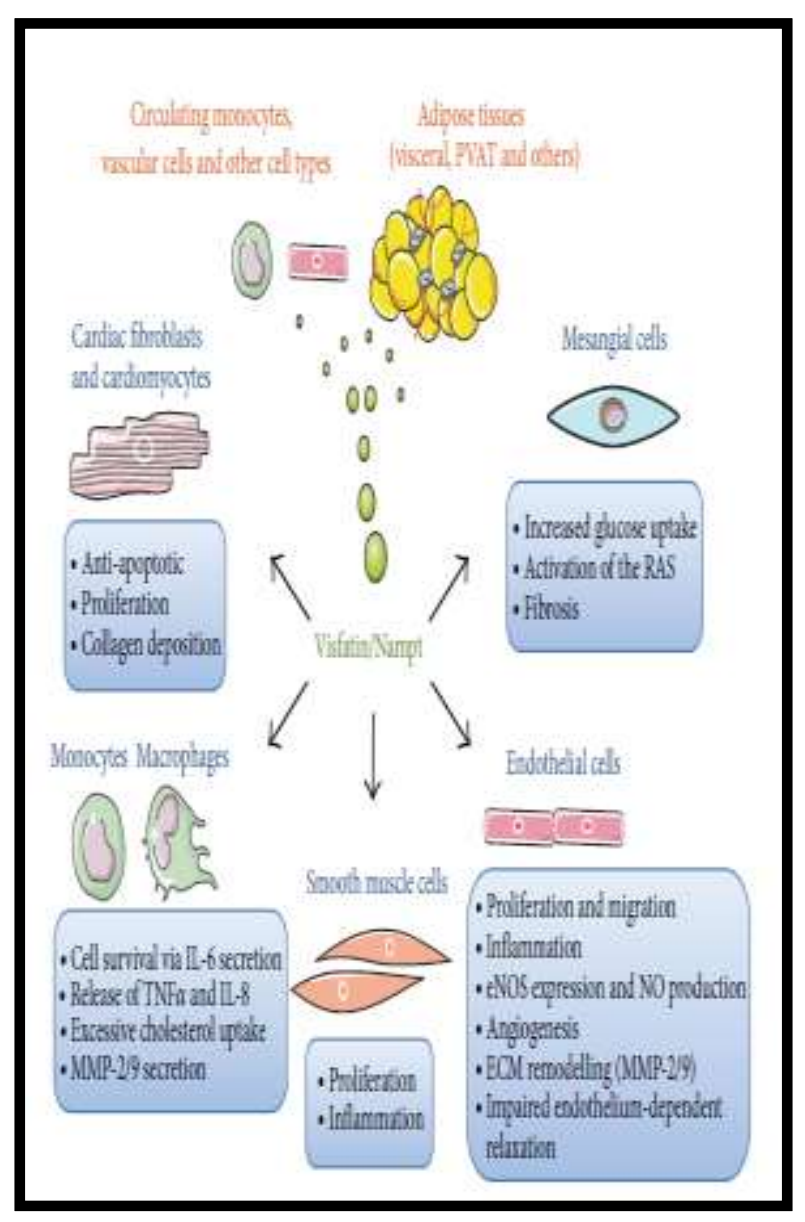

Figure (3): Diagram summarizing the main reported direct actions of visfatin/Nampt in cells in the cardiovascular system, adapted by (Romacho et al., 2013)

\section{ACKNOWLEDGMENT}

Not applicable. 


\section{REFERENCES}

Adya, R.; Tan, B.; Punn, A.; Chen, J. and Randeva, H. (2008).Visfatin induces human endothelial VEGF and MMP-2/9 production via MAPK and PI3K/Akt signalling pathways: novel insights into visfatin-induced angiogenesis. Cardiovascular Research, 78 (2):356-365, 2008.

Bae, Y.; Bae, M.; Kim, S.; Lee, J.; Wee, H. and Bae, S. (2009). Upregulation of fibroblast growth factor-2 by visfatin that promotes endothelial angiogenesis. Biochemical and Biophysical Research Communications, 379.(2): 206-211.

Costford, S.R.; Bajpeyi, S.; Pasarica, M.; Albarado, D.C.; Thomas, S.C.; Xie, H. and et al.(2010). Skeletal muscle NAMPT is induced by exercise in humans. American Journal of Physiology -Endocrinology and Metabolism, 298(1): 117-26.

Curat , C.A.; Wegner, V.; Sengenes, C,.; Miranville, A.; Tonus, C.; Busse, R. and et al. (2006). Macrophages in human visceral adipose tissue: increased accumulation in obesity and a source of resistin and visfatin. Diabetologia, 49(4): 744-747.

Dahl, T.; Yndestad, A.; Skjelland, M. and et al.(2007). Increased expression of visfatin in macrophages of human unstable carotid and coronary atherosclerosis: possible role in inflammation and plaque destabilization. Circulation, 115(8) : 972980.

De-Clercq, V.; Taylor, C. and Zahradka, P.(2008). Adipose tissue: the link between obesity and cardiovascular disease. Cardiovasc. Hematol. Disord. Drug Targets,8:228-37.

Dragovich, P.; Zhao, G.; Baumeister T.; Bravo, B. and Giannetti, A.(2014). Fragment-based design of 3aminopyridine-derived amides as potent inhibitors of human nicotinamide phosphoribosyltransferase (NAMPT). Bioorganic \& Medicinal Chemistry Letters, 24, (3): 954-962.

Filippatos, T.D.; Randeva, H.S.; Derdemezis, C.S.; Elisaf, M.S. and Mikhailidis, D.P. (2010). Visfatin/PBEF and atherosclerosis-related diseases. Curr. Vasc. Pharmacol., 8:12-28.

- Filippatos, T.D.; Tsimihodimos, V.; Derdemezis, C.S.; Gazi, I.F.;Saougos, V.; Mikhailidis, D.P. and et al. (2013). Increased plasma visfatin concentration is a marker of an atherogenic metabolic profile. Nutr. Metab. Cardiovasc. Dis., 23:330-6.

Flier, J.s.; Flier, E.m., Wiener, C.; Fauci A.S. and et al. (2007). Biology of Obesity. In: Harrison's Principles of Internal Medicine . The Mcgraw-Hill companies Inc, New York, pp. 462- 472.

Fukuhara, A.; Matsuda, M.; Nishizawa, M.; Segawa, K.; Tanaka, M. and et al. (2005). Visfatin: a protein secreted by visceral fat that mimics the effects of insulin. Science, 307: 426430.

Garten, A.; Petzold, S.; Barnikol-Oettler, A.; Korner, A.; Thasler, W.E.; Kratzsch, J. and et al. (2010). Nicotinamide phosphoribosyltransferase (NAMPT/PBEF/visfatin) is constitutively released from human hepatocytes. Biochemical and Biophysical Research Communications, 2010;391(January (1)):376-81.

Gürsoy, M.; Duygu, E.; Hökenek, A.F.; Gülcan, F. and Kınoğlu, B. (2014). Serum Visfatin Levels and Coronary Artery Disease. Koşuyolu Heart Journal, 17(2):95-99.

Jia, S.H.; Li, Y.; Parodo, J.; Kapus, A.; Fan, L.; Rotstein, O.D. and et al.(2004). Pre-B cell colonyenhancing factor inhibits neutrophil apoptosis in experimental inflammation and clinical sepsis. Journal of Clinical Investigation, 113(9):1318-27.

Kim, M.k.; Lee, J.h.; Kim, H.; Park, S.j.; Kim, S.h.; Kang, G.b. al., (2006). Crystal structure of visfatin/pre-B cell colony-enhancing factor $1 /$ nicotinamide phosphoribosyltransferase, free and in complex with the anti-cancer agent FK-866. Mol. Biol., 362: 66-77.

Kitani, T.; Okuno, S. and Fujisawa, H. (2003). Growth phasedependent changes in the subcellular localization of pre-B-cell colony-enhancing factor. FEBS. Lett., 544:74-8.

Lee, W.; Wu, C.; Lin, H. and et al. (2009). Visfatininduced expression of inflammatory mediators in human endothelial cells through the NF-kappaB pathway. International Journal of Obesity, 33 (4):465-472.

Li, Y.; Zhang, Y.; Dorweiler, B. and et al.(2008). Extracellularnampt promotes macrophage survival via a nonenzymatic interleukin- 6/STAT3 signaling mechanism. Journal of Biological Chemistry, 283(50):34833-34843.

Matsuda, F. and Nishizawa, M. et al. (2005). Visfatin: a protein secreted by visceral fat that Mimics the effects of insulin. Sci., 2005; 307:426-30.

Mazaherioun, M.; Hosseinzadeh-Attar, M.J.; Janani, L.; Vasheghani Farahani, A.; Rezvan, N.; Karbaschian, Z. and et al. (2012). Elevated serum visfatin levels in patients with acute myocardial infarction. Arch. Iran Med., 15(11): 688 - 692.

Moschen, A. .; Kaser, A.; Enrich, B. and et al. (2007). "Visfatin, an adipocytokine with proinflammatory and immunomodulating properties. Journal of Immunology, 178(3): 1748-1758.

Moschen,. AR.; Gerner, R.R. and Tilg, H. (2010). Pre-B cell colony enhancing factor in inflammation and obesity -related disorders. Curr. Pharm. Des., 16(17):1913-20. 
Moulton, K.S.(2006). Angiogenesis in atherosclerosis: gathering evidence beyond speculation. Current Opinion in Lipidology, 17(5):548-555.

Naveen, G. (2009). A study of lipid profile in smokers and it is association in ischemic heart disease, Dissertation, M.D. (General Medicine), P39.

Olszanecka, G.; Linianowicz, M.; Kocelak, P.; Janowska, J.; Skorupa, A.; Nylec, M. and Zahorska, M. (2011). Plasma visfatin and tumor necrosis factor -alpha level in metabolic syndrome. Kardiol. Pol., 69(8):802-7.

Omer, R. and Mahmood, M. (2016). Correlation between Visfatin and Creatine Kinase Levels with Periodontal Health Status of Patients with Coronary Atherosclerosis and Chronic Periodontitis. (J. Bagh. Coll. Dentistry, 28(3):121125)

Raman, T. (2013). Adipokines, structure of visfatin. Health \& Medicine, Technology J.

Romacho, T.; Azcutia, V.; Vazquez-Bella, M.; Matesanz, N.; Cercas, E. and et al. (2009). Extracellular $\mathrm{PBEF} / \mathrm{NAMPT} / \mathrm{visfatin}$ activates proinflammatory signalling in human vascular smooth muscle cells through nicotinamide phosphoribosyltransferase activity. Diabetologia. 52 (11)2455-2463.

Romacho, T.; Sánchez-Ferrer, C. and Peiró, C. (2013). Visfatin/Nampt: An Adipokine with Cardiovascular Impact. Mediators of Inflammation, 15 pages.

Rydén, L.; Standl. E.; Bartnik, M.; Van den Berghe, G.; Betteridge J.; de Boer. M.J.; Cosentino, F. and Jönsson, B. (2007). Guidelines on diabetes, prediabetes, and cardiovascular diseases. Eur. Heart J., 28, 88-136.

Samal, B.; Sun, Y.; Stearns, G.; Xie, C.; Suggs, S. and McNiece, I. (1994). Cloning and characterization of the cDNA encoding a novel human pre-B-cell colony-enhancing factor. Molecular and Cellular Biology, 14 (2):1431-7.

Sebregts, E.H.; Falger, P.R and Bar, F.W. (2000). Risk factor modification through non-pharmacological interventions in patients with coronary heart diseases. Journal of Psychosomatic Research, 48: 425- 41 .

Shaker, O.; El-Shehaby, A.; Zakaria, A.; Mostafa, N.; Talaat, S.; Katsiki, N. and et al.(2011). Plasma visfatin and retinol binding protein-4 levels in patients with type 2 diabetes mellitus and their relationship to adiposity and fatty liver. Clin. Biochem., 44:1457-63.

Stofkova, A. (2010). Resisiten and visfatin: regulators of insulin sensitivity, inflammation and immunity. Endocr. Regul., 44: 25-36.

Trivedi, D. and Chhaya, T. (2012). Salivary proteome in periodontal diagnosis. International. J. Pharma. and Bio.Sci., 2:5-9.

Wang, L.S.; Yan, J.J.; Tang, N.P.; Zhu, J.; Wang, Y.S. and et al., (2011). polymorphism in the visfatin gene promoter is related to decreased plasma levels of inflammatory markers in patients with coronary artery disease. Mol. Biol, Rep., 38(2): 819-25.

Wang, P.; Xu, T.; Guan, Y.; Su, D.; Fan, G. and C. Miao, C. (2009). Perivascular adipose tissue-derived visfatin is a vascular smooth muscle cell growth factor: role of nicotinamide mononucleotide, Cardiovascular Research, 81 (2): 370-380.

Wang, T.; Zhang, X.; Bheda, P.; Revollo, J.r.; Imai, S. and Wolberger, C. (2006). Structure of Nampt/PBEF/visfatin, a mammalian NAD+ biosynthetic enzyme. Nat. Struct. Mol. Biol. 13: 661-662. 